\title{
Apoptosis in Greece
}

\author{
Sergio A. Lamprecht ${ }^{1}$ and John D. Delinassios ${ }^{2}$ \\ ${ }^{1}$ Gastroenterology Department, Soroka Medical Center and Department of Clinical Biochemistry, Ben-Gurion University of the Negev, Beer Sheva, Israel \\ ${ }^{2}$ International Institute of Anticancer Research, Athens, Greece
}

\section{International Conference on Apoptosis}

Athens, Greece, October 2-5, 1997

\begin{abstract}
Abbreviations: TGF- $\beta$, transforming growth factor-beta; PKC, protein kinase C; NSAIDS, Nonsteroidal anti-inflammatory drugs; COX, cyclooxygenase; GI, gastrointestinal; Bcl-2, B-cell lymphoma/leukemia-2 protein; EM, electron microscopy; ROS, reactive oxygen species; $\mathrm{Bax}, \mathrm{Bcl}-2$-associated $\mathrm{x}$-protein; $\mathrm{NF} \kappa \mathrm{B}$, nuclear factor $\kappa \mathrm{B}$; IIAR, International Institute of Anticancer Research; SSCP, single-strand conformational polymorphism
\end{abstract}

The International Conference on Apoptosis was held in the International Institute of Anticancer Research (Athens, Greece) from October 2-5 1997. The program consisted of main lectures, short talks, a session devoted to research aims and poster presentations, all of which covered a wide interdisciplinary spectrum of apoptosis research. Here we survey the salient features of the conference. No attempt was made to cover all the speakers and poster sessions.

The session devoted to the biochemical mechanisms of apoptosis began with an incisive talk by $\mathrm{S}$. Dormann (Freiburg, Germany). The Freiburg team, headed by G. Bauer, has provided vast evidence that TGF- $\beta$ synthesized by transformed fibroblasts triggers nontransformed homotypic cells to release a short-lived apoptosis-inducing factor (or factors) specifically directed against transformed cells. Dormann elegantly discussed the distinct but interrelated roles of TGF- $\beta$ in the lethal cell dialogue. A most interesting observation was that TGF- $\beta$ causes downmodulation of endogenous survival factors in the transformed cells. This finding not only re-emphasizes the importance of context in defining TGF- $\beta$ pleiotropic actions, but also proposes a novel mechanism of apoptosis induction.

A. Dvilansky (Beer Sheva, Israel) presented solid evidence that in a number of human leukemic cell lines the antitumoral growth-restraining action of non-steroidal antiestrogens (e.g., nafoxidine) is associated with the induction of apoptosis. The effects of the drugs was abrogated by antioxidants. Data were shown suggesting a contributory role of PKC in the apoptotic process.

Of late, much attention is focused on the role of mitochondria in the apoptotic suicide pathway. P. Schotte (Ghent, Belgium) presented convincing findings indicating that the decrease of mitochondrial transmembrane potential releases a factor able to induce apoptosis in isolated nuclei. The apoptogenic mitochondrial agent triggers the processing of caspase 3 and caspase 11 with subsequent activation of the ICE-like proteases. These data substan- tiated by an elegant series of experiments strengthen the recurring observation of an intimate relationship between mitochondria and the caspase system in apoptosis. The results indicate that caspases' activity is located downstream to the mitochondrial apoptotic response.

Intense interest is centered on non-steroidal antiinflammatory drugs such as aspirin and sulindac as chemopreventive agents against colorectal cancer. Convincing evidence is available showing that these drugs induce apoptosis in colonic cells. D.J.E. Elder (Bristol, U.K.) reviewed incisively the present knowledge pertaining to the NSAIDs-COX system. He reported findings on the inducible form COX-2 which, while promoting colon carcinogenesis, is inhibited by NASAIDs with very low GI toxicity. The effect of the COX-2 selective inhibitor NS-398 was examined on two colorectal carcinoma cell lines, one expressing constitutively COX-2 (HT-29), the other apparently devoid of enzyme activity (S/KS). Both lines do not express wild-type p53. The results clearly expounded showed that NS-398 induces apoptosis in both cell lines. This action was p53-independent and surprisingly did not require the presence of the COX-2 protein. How then does the COX-2 inhibitor exert its apoptotic, antineoplastic action?

The afternoon session pertaining to cellular and molecular biology of apoptosis began with an introductory lecture by S. Lamprecht (Beer Sheva, Israel) aptly entitled 'apoptosis alive today.' Lamprecht examined in detail our present understanding of the apoptosis process trying to identify a central, nodal step of no-return where ineluctable execution of apoptosis takes place, irrespective of the disparate initial stimuli, whether stochastic or determined. Inevitably this inquiry led to an examination of the caspase family. He argued that the promiscuous presence of the caspase zymogens in nuclei, cytoplasm and possibly in mitochondria is too perilous, with the stage set for an accidental and catastrophic amplifying cascade. We therefore miss some critical information on stringent regulatory devises which normally prevent unscheduled caspase activation.

Lamprecht also expressed doubts as to whether the life and death of a cell simply relies on a stoichiometic relationship between various proteins of the bcl-2 family, finding this control mechanism, albeit economical for the cell, too 'coarse' for determining the cell fate. In a separate talk, he also presented recent findings from his laboratory 
(Lifshitz) based on an ex-vivo model for harvested colonic cells from the crypt continuum. The cells deprived of crypt habitat activated an intrinsic death program irrespective of their position along the crypt axis at harvest time. Attenuation of tyrosine phosphorylation was associated with the apoptotic process in colonic cells, strengthening the recurring observation that post-translation events play a determining role in the execution of apoptosis.

In the same session, H. Zoellner (Sydney, Australia) discussed intriguing findings pertaining to the inhibition of endothelial apoptosis by serum albumin in rat skin organ culture. Apoptosis was assessed both by counting of apoptotic nuclei and confirmed by EM. Importantly, neither bovine serum albumin nor ovoalbumin were effective in protecting cultured endothelial cells from apoptosis, suggesting a specific role of human albumin in this context. The lecturer suggested a determining role for albumin in modulating the vascular form via regulation of endothelial apoptosis, an novel action of albumin not less important than the 'traditional' functions attributed to the protein.

C. Humblet (Liege, Belgium) presented convincing evidence that after leukemogenic irradiation of mice there is an increase in thymic cells wherein the suicide program is activated; however, environmental thymic factors rescue them from apoptosis. The interesting proposition was made that preleukemic cells are part of this abnormally surviving population of cells 'progammed to die' which, in time, acquire additional genomic alterations leading to the full neoplastic phenotype.

In the session pertaining to apoptosis and oncogenesis, G. Bauer (Freiburg, Germany) presented an incisive and intellectually-demanding lecture entitled: 'Control of oncogenesis by intercellular induction of apoptosis.' A fascinating story emerged whereby cross-talk between transformed cells and normal neighbors leads ultimately to the demise of the cancer cells by apoptotic death and their elimination from the healthy cell social fabric. Convincing findings showed that TGF- $\beta$ released from transformed fibroblasts triggers homotypic cells to release a short-lived apoptosis factor specifically directed against the transformed cells. ROS species are involved in the process and p53 plays no role in the induction of apoptosis. Interestingly, the apoptotic events in transformed fibroblasts are associated with inactivation of endogenous survival signals. The last concept was expounded further in a talk by A. Schwieger from Bauer's group in Freiburg. Albeit evidence for the involvement of survival factors in fibroblastic co-culture remains circumstantial, the speaker discussed with clarity an interesting model whereby downregulation of putative endogenous survival factors permits efficient induction of apoptosis in transformed cells. Kupryanczynk (Warsaw, Poland) presented robust findings suggesting that the p53 status, characterized by immunohistochemical and SSCP procedures followed by sequencing of p53 variants, does not affect the apoptosis rate in ovarian carcinomas prior to chemotherapy.

The next two sessions were focused on the genetic regulation of apoptosis. In an clear and precise talk, Madireddi (New York, USA) presented rigorous experi- mental evidence for the isolation by subtraction hybridization of a novel tumor suppressor gene (mda-7) in differentiation-induced human melanoma cells. Mda-7 encodes a novel polypeptide with a predicted size of 23.8 $\mathrm{kDa}$. Transfection experiments of an mda-7 expression construct into disparate cancer cells indicated that the coded peptide is able to restrain cell growth. Interestingly, overexpression of the gene in various human breast adenocarcinoma cell lines provoked DNA fragmentation and was accompanied by the unequivocal induction of the apoptosis agonist protein Bax. Of note, normal breast cell lines were refractory to the apoptosis-inducing action of mda-7. The potential therapeutic usefulness of the novel gene in the treatment of human breast tumors is evident. $\mathrm{H}$. Eichholtz-Wirth (Munchen, Germany) reported that drug resistance in HeLa cells is associated with a diminished number of annexin-positive cells and reduced levels of caspase proteins, and elaborated on APO-I/Fas and NFkBassociated changes in caspase 3-dependent apoptotic pathway of radiation-induced cisplatin resistant HeLa cells. G. Tsangaris (Athens, Greece) presented an incisive talk pertaining to regulation of apoptosis of blasts cells in peripheral blood and bone marrow in acute leukemia. The immunohistochemical expression of a number of genes (p53, c-myc, fas, mdr-I and bcl-2) was evaluated in bone marrow and peripheral blood smears of children with newly diagnosed leukemia. The interesting conclusion was reached that in vivo the rapid stimulation of chemotherapy-induced apoptosis depends on p53, c-myc or fas expression while interference with the apoptotic process associated with resistance to chemotherapy required $\mathrm{mp} 53$ (a p53 mutant form), bcl-2 and mdr-1.

Tsangaris, who has developed a versatile and elegant nuclear staining method for identification of apoptosis in peripheral blood samples, convincingly integrated the wealth of results providing valuable information about the regulation of chemotherapy-induced apoptosis, the response of patients to treatment and relapse prediction. In a subsequent talk, this speaker reported that the use of antisense oligonucleotides directed against human metallothionein transcripts prevents apoptosis in an human immature $\mathrm{T}$ cell line. This observation is intriguing since it proposes that one of the functions of metallo thioneins is to provide 'a cellular protective mechanism neutralizing external transient apoptotic signals'.

The final sessions were devoted to apoptosis in cancer therapy. V.I. Avramis (Los Angeles, USA) presented a wellordered talk showing that treatment with synergistic antileukemic drug is associated with upregulation of p53, p21 and downregulation of bcl-2 in human leukemic cells. A sequence of events was delineated whereby the drug treatments produce high m.wt. DNA fragmentation, followed by upregulation/stabilization of p53 and p21 WAF-1 proteins which, in turn, inhibit cyclin-dependent kinases triggering the internucleosomal DNA cleavage and the final cell demise.

The bcl-2 oncoprotein when overexpressed in prostate cancer cells renders the cells resistant to a spectrum of therapeutic agents, including hormonal ablation. This provided the rationale for an elegant series of experiments 
Meeting Report

based on ribozome technology described with competence by $\mathrm{T}$. Dorai (New York, USA). An anti-bcl-2 ribozyme (RZ) able to catalytically destroy bcl-2 mRNA was directly transfected into cultured prostate LNCaP cancer cell variants. $\mathrm{Bcl}-2$ mRNA levels were measured by a ribonuclease protection assay following culture conditions conductive to apoptosis, i.e. serum starvation or frank apoptotic stimulus (TPA). The results suggest that prior treatment with the anti-bcl-2 ribozyme sensitizes the high bcl-2 expressing hormone resistant prostate cancer cells to the subsequent action of disparate apoptotic agents. R.J. Stewart (Randwick, Australia) expounded in a precise and well-construed talk findings on the analysis of etoposideinduced apoptosis in human leukemic cells. Starting with the important, often ignored, remark that apoptotic response to drugs is often asynchronous and thereby elucidation of distinct temporal changes in the apoptotic cascade is consequently blurred, the speaker presented data related to CEMor MOLT-4 lymphoblastoid cells induced to die synchronously in the presence of low concentrations of etoposide. Under these conditions, earlier responses to drug treatment included expression of genes controlling lymphocytes maturation, overexpression and activation of caspase 3. Subsequently, G2/M cell cycle arrest and $50 \mathrm{~kb}$ DNA fragmentation was noted 'upstream' to the stereotyped ultrastructural changes observed in apoptotic cells.

In conclusion, the IIAR Conference on Apoptosis was characterized by a wide interdisciplinary approach to the scrutiny of the apoptotic process. The scientific standard of lectures and posters was high. Many senior participants were accompanied by their students who presented with youthful elan research works in progress. The location of the IIAR Institute, an elegant white building in the middle to the gentle hilly Greek countryside, the gracious and urbane hospitality, and the small number of participants made possible an informal and relaxed atmosphere where alive discussion was easily promoted. This was clearly evident in the session related to future principal research aims in apoptosis. The consensus was that considerable caution must be exercised in the interpretation of vitro results in view of evidence derived from knockout mice, pointing to much redundancy and tissue-specificity of the apoptosis machinery.

Final Remarks: To maintain objectivity, J.D.D. wrote the section pertaining to S.A.L. The conference was supported by the Ministry of Development, Greece, the International Institute of Anticancer Research, Athens, Greece and the International Society for the Study of Comparative Oncology, Maryland, USA (President: Prof. H.E. Kaiser). 